\title{
Short fatigue crack growth in welded joints described by the effective cyclic J-integral
}

\author{
Désiré Tchoffo Ngoula ${ }^{1, *}$, Michael Vormwald ${ }^{1}$ \\ ${ }^{1}$ Materials Mechanics Group, Technische Universität Darmstadt, Franziska-Braun-Str. 3, 64287 Darmstadt, Germany
}

\begin{abstract}
The purpose of the present contribution is to predict the fatigue life of welded joints by using the effective cyclic $J$-integral as crack driving force. The plasticity induced crack closure effects and the effects of welding residual stresses are taken into consideration. Here, the fatigue life is regarded as period of short fatigue crack growth. The node release technique is used to perform finite element based crack growth analyses. For fatigue lives calculations, the effective cyclic $J$-integral is employed in a relation similar to the Paris (crack growth) equation. For this purpose, a specific code was written for the determination of the effective cyclic $J$-integral for various lifetime relevant crack lengths. The effects of welding residual stresses on the crack driving force and the calculated fatigue lives are investigated. Results reveal that the influence of residual stresses can be neglected only for large load amplitudes. Finally, the predicted fatigue lives are compared with experimental data: a good accordance between both results is achieved.
\end{abstract}

\section{Introduction}

Welding is one of the most important joining methods in technology, particularly for metallic structures. Thus, improving the design of welded structures is of high technical use. Welded structures are generally exposed to fatigue failure: under cyclic external loading, an immense part of the crack growth takes place in the weld notch area. As a result of the notch effect, the local behaviour of the weld in this area might be elasticplastic. Thereby, the material is subjected to transient processes like the cyclic hardening/softening, the cyclic relaxation and ratcheting.

During the welding process, residual stresses can arise in the joint due to the heating and cooling cycles, which belongs to this process. In case of an external cyclic loading, these stresses can have an influence on the fatigue life of the welded structure: the presence of a tensile residual stress field at the failure critical notch for example reduces the fatigue life, while a compressive residual stress field extends it $[1,2]$.

Crack closure effects were observed for the first time by Elber [3]. During cyclic loading, crack closure means that the two crack faces contact each other during the unloading phase of a cycle before the minimum load is reached, or that the crack remains closed during the loading phase of a cycle until a certain load level [4]. Therefore, only the load portion, in which the crack is open, is responsible for the propagation of the crack (keyword "effective load"). Closure effects lead to a reduction of the crack driving force and may influence the crack propagation rate. Therefore, to avoid significant errors in the fatigue life assessment of welded joints, residual stresses and crack closure effects should be considered.

Different approaches can be used to assess the fatigue life of welded joints: the nominal stress approach, the structural stress approach, the notch stress approach, the notch strain approach and the crack propagation approach $[5,6]$. The main different between these approaches is the parameter employed for the description of the fatigue life. Many studies [7] have shown that, the lifetime estimation of welded joints can be done by using the fracture mechanics concept (crack propagation approach) alone, because the crack nucleation stage is very short (due to the presence of defects in the weld) and can be neglected compared to crack propagation stages. Thus, the main part of the fatigue life falls on the crack propagation, precisely on the short crack growth [8]. Accordingly, the application of the elastic-plastic fracture mechanics will be required because the length of the plastic zone at the crack tip (in case of short cracks) is not negligible, compared to the crack length.

For the modelling of fatigue crack growth in elasticplastic fracture, Dowling and Begley [9] proposed to use the cyclic $J$-integral, $\Delta J$, as crack driving force. $\Delta J$ is an extension of the monotonic $J$-integral introduced by Rice [10]. This parameter has successfully been applied as an appropriate crack driving force for the description of fatigue crack growth, when the elastic-plastic material exhibits Masing's behaviour [11,12]. $\Delta J$ can be related to the increase in crack length per cycle, $\mathrm{d} a / \mathrm{d} N$, by the following power-law expression:

$$
\frac{\mathrm{d} a}{\mathrm{~d} N}=\mathrm{C}_{\mathrm{J}} \cdot(\Delta J)^{\mathrm{m}_{\mathrm{J}}}
$$


where, $\mathrm{C}_{\mathrm{J}}$ and $\mathrm{m}_{\mathrm{J}}$ are material dependent parameters which can be determined experimentally.

The purpose of this work is to calculate the fatigue life of welded joints by taking into account plasticity induced crack closure effects as well as the effect of welding residual stresses. Here, the fatigue life is regarded as period of short fatigue crack growth. Analyses are performed with two and three dimensional finite element models, by using the crack propagation approach. Closure effects were considered by defining contact boundary conditions between crack faces and by using the effective cyclic $J$-integral $\left(\Delta J_{\text {eff }}\right)$ as crack tip parameter for the description of the crack propagation rate. The advantage of this approach (fracture mechanics concept) is that, residual stresses can be treated explicitly. However, the numerical determination of $\Delta J_{\text {eff }}$ appeared to be complex. A specific code was written for the determination of $\Delta J_{\text {eff }}$ at each crack length configuration.

The research results in this contribution were achieved at the Materials Mechanics Group of Technische Universität Darmstadt in the scope of a project called IBESS ("Integrale Bruchmechanische Ermittlung der Schwingfestigkeit von Schweißverbindungen" [13]). The aim of IBESS is the fracture mechanics based simulation of Wöhler curves (S-N curves) in welded joints. IBESS is divided into 8 subprojects and the authors were working on the subproject titled: "Modelling of fatigue crack growth in welded joints under consideration of the transient plastic deformation behaviour."

\section{Geometry and material}

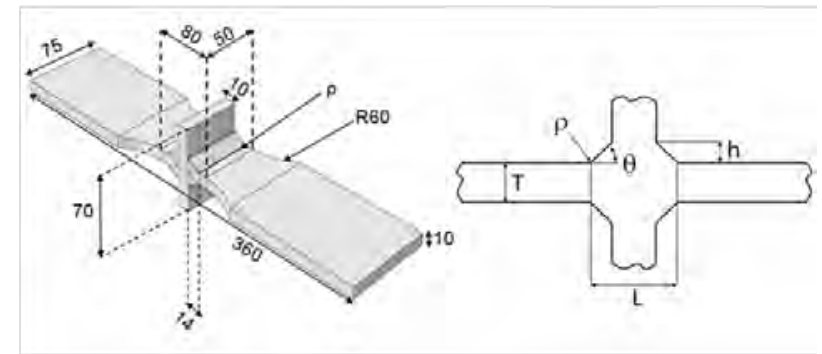

Fig. 1. Geometry and sizes (in $\mathrm{mm}$ ) of the cruciform welded joint examined [14].

The structure used in this contribution is a cruciform welded joint. In this case, it is a full penetration weld. The geometry and dimensions of the structure are shown in Fig. 1. This specimen has a thickness T of $10 \mathrm{~mm}$ and a length of about $360 \mathrm{~mm}$. The weld width $\mathrm{L}$ is about 38 $\mathrm{mm}$ and the investigated cross section is $50 \mathrm{~mm} \times$ $10 \mathrm{~mm}$, i.e $500 \mathrm{~mm}^{2}$. Other sizes can be found in the figure.

The weld toe radius $\rho$ and the weld flank angle $\theta$ are parameters which can scatter. They were measured and documented by a project partner $[15,16]$. According to the project partner, $\rho$ has a mean value of approximately $1 \mathrm{~mm}$ and varies from $0,2 \mathrm{~mm}$ to $3,0 \mathrm{~mm}$. In addition, $\theta$ varies between 3 and 40 degrees.
The tested specimens were made from medium strength structural steel S355NL. The cyclic material data for the Ramberg-Osgood equation,

$$
\varepsilon=\varepsilon_{e l}+\varepsilon_{p l}=\frac{\sigma}{E}+\left(\frac{\sigma}{K^{\prime}}\right)^{\frac{1}{n^{\prime}}},
$$

were determined by a project partner (IEHK, RWTH Aachen): $\quad K^{\prime}=1115 \mathrm{MPa}, n^{\prime}=0,1961$ and $E=$ $210000 \mathrm{MPa}$. By using these cyclic material data, the Chaboche's material [17] parameters were also determined:

$$
\sigma=\sigma_{y}+\sum_{i=1}^{5}\left[\frac{C_{i}}{\gamma_{i}}\left(1-\exp \left(-\gamma_{i} \varepsilon_{p l}\right)\right)\right]
$$

These parameters (the coefficients $C_{i}, \gamma_{i}$ as well as the yield stress $\sigma_{y}$ ) are summarized in Table 1 and were used in FE calculations.

Table 1. Chaboche's material parameters of steel S355NL.

\begin{tabular}{|lllclc|}
\hline$i$ & \multicolumn{1}{c}{1} & \multicolumn{1}{c}{2} & \multicolumn{1}{c}{3} & \multicolumn{1}{c}{4} & 5 \\
\hline$C_{i}[\mathrm{MPa}]$ & 305034 & 98322 & 90427 & 26762,3 & 7607 \\
$\gamma_{i}$ & 12318,92 & 3654,02 & 1204,24 & 230,50 & 21,07 \\
$\sigma_{y}[\mathrm{MPa}]$ & 152,17 & & & & \\
\hline
\end{tabular}

\section{Calculation of the cyclic J-integral}

Under non-monotonic loading, the $\Delta J$-integral proposed by Dowling and Begley [9] is generally used in the framework of elastic-plastic fracture mechanics as crack tip parameter and is defined as:

$$
\Delta J=\int_{\Gamma}\left(\Delta W d x_{2}-\Delta t_{i} \frac{\partial \Delta u_{i}}{\partial x_{1}}\right) d s
$$

with

$$
\Delta W=\int_{0}^{\Delta \varepsilon_{i j}} \Delta \sigma_{i j} \mathrm{~d} \Delta \tilde{\varepsilon}_{i j}, \quad \Delta t_{i}=\Delta \sigma_{i j} n_{j}
$$

and with

$$
\Delta \sigma_{i j}=\sigma_{i j}-\sigma_{i j}^{0}, \Delta \varepsilon_{i j}=\varepsilon_{i j}-\varepsilon_{i j}^{0}, \Delta u_{i}=u_{i}-u_{i}^{0} .
$$

Thereby, $\Gamma$ is a crack tip enclosing path, $d s$ is a small element on $\Gamma, W$ is the deformation energy density, $t_{i}$ is the stress vector on the path $\Gamma, u_{i}$ is the displacement vector of the path's points and $n_{j}$ is a local outward normal vector to $\Gamma . \Delta \sigma_{i j}, \Delta \varepsilon_{i j}$ and $\Delta u_{i}$ are respectively the ranges of stresses, strains and displacements. The index 0 in Eq. (6) symbolizes an initial state which is the state of the structure at load reversal. Note that $\Delta J$ and $\Delta W$ are not ranges (maximum value minus minimum value in a cycle), but absolute values. By assuming the deformation plasticity, $\Delta J$ is like $J$ also path independent and can therefore be used as crack tip parameter.

The integration of Eq. (4) must be done with a postprocessing code after finite element calculations have been performed. This means that, at every step increment, the finite element results for stresses, strains 
and displacements must be available in the area of interest. First of all, the integration path $\Gamma$ must be defined and the local outward normal vectors, $n_{j}$ (Eq. 8), at its points must also be calculated. Secondly, the integration limits of Eq. (4) are to be defined. This integration can be done from the lower load reversal point to the upper one or vice-versa (if closure effects are not considered).

The trapezoidal rule is used for the determination of $\Delta W$ (cyclic counterpart of the strain energy density):

$$
\Delta W \approx \sum_{L=L_{\text {lower }}}^{L_{\text {upper }-1}}\left[\frac{\Delta \sigma_{i j}^{L+1}+\Delta \sigma_{i j}^{L}}{2} \cdot\left(\Delta \varepsilon_{i j}^{L+1}-\Delta \varepsilon_{i j}^{L}\right)\right]
$$

Thereby, $L$ is the load increment counter, $L_{\text {lower }}$ and $L_{\text {upper }}$ symbolize the lower and upper load reversal points respectively, which are the integration limits in this case. The components of the local stress vector range, $\Delta T_{i}$, are calculated at every load increment by using the following relationship:

$$
\Delta T_{i}^{L}=\Delta \sigma_{i j}^{L} \cdot n_{j}=\left(\sigma_{i j, \max }^{L}-\sigma_{i j, \min }^{L}\right) \cdot n_{j}
$$

The partial derivative of the local displacement vector, $\frac{\partial \Delta u_{i}}{\partial x}$, is approximated with difference quotients. To do this, the path $\Gamma$ is to be shifted left (negative direction, index 1 in Eq. (10)) and right (positive direction, index 2 in Eq. (10)) along the $\mathrm{x}$-axis (parallel to the crack line) by an amount of $\delta x / 2$ :

$$
\frac{\partial \Delta u_{i}^{L}}{\partial x} \approx \frac{\Delta u_{i}^{L,(2)}-\Delta u_{i}^{L,(1)}}{\delta x}
$$

where

$$
\Delta u_{i}^{L,(1)}=\left.\Delta u_{i}^{L}\right|_{\Gamma-\frac{\delta x}{2}}, \Delta u_{i}^{L,(2)}=\left.\Delta u_{i}^{L}\right|_{\Gamma+\delta x / 2}
$$

Besides, the previously determined auxiliary variable, $\delta x$, is to be chosen very small. Eq. (4) can now be evaluated completely.

\subsection{Effective cyclic J-integral}

If crack closure effects are to be considered, the $\Delta J$ integral is replaced by the effective $\Delta J$-integral, $\Delta J_{\text {eff }}$. Equation (4) is utilized for the calculation of $\Delta J$, as well

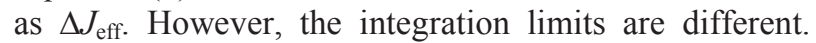
The integration limits for $\Delta J$ are the load reversal points whilst $\Delta J_{\text {eff }}$ is computed during the unloading stage of a cycle and the integration limits are respectively the upper load reversal point and the crack closure time.

In order to capture crack closure effects $[12,18,19]$ in the FE-model, contact boundary conditions have to be defined between crack faces. The contact opening displacements of all the crack face nodes were monitored during the cyclic loading for the determination of the crack closure time, $t_{c l}$. The crack is considered to be closed if one of these contact opening displacements becomes zero during the unloading stage of a cycle.
Post-processing codes were written to determine $\Delta J$ and $\Delta J_{\text {eff }}$ as function of the crack length/depth for $2 \mathrm{D}$ as well as $3 \mathrm{D}$ geometry [20]. It was possible to evaluate $\Delta J$ and $\Delta J_{\text {eff }}$ numerically at each crack length configuration. Note that, different to $J$, the estimation of $\Delta J$ and $\Delta J_{\text {eff }}$ cannot be performed directly in a finite element program.

\subsection{Path-independency of the cyclic J-integral}

A 3D model of a cruciform joint was used to study the path-independency of $\Delta J$. A semi-circular surface crack was placed at one of the weld toes, such that there is symmetry with respect to the mid-plane (perpendicular to the welds length direction). By exploiting this symmetry plane, only half of the investigated structure needs to be modelled (see Fig. 2). The commercial finite element program ABAQUS was used for the modelling. The upper part of Fig. 2 is a detail of the FE-model which shows the prescribed crack geometry. The maximum element length in the predefined crack area is $0,05 \mathrm{~mm}$. Elements with linear shape functions were applied. The specimen was loaded with a cyclic nominal stress $S$ in the direction perpendicular to crack faces ( $x$ direction). Closure effects were not considered and the node release technique $[12,19]$ was used for the gradual extension of the crack. The ratio $a / c=1$ was kept constant during node release. The increase in crack depth $\Delta a$ was chosen to be $0,05 \mathrm{~mm}$ and only two load cycles were applied for every crack length configuration in order to restrict the FE-calculation time.
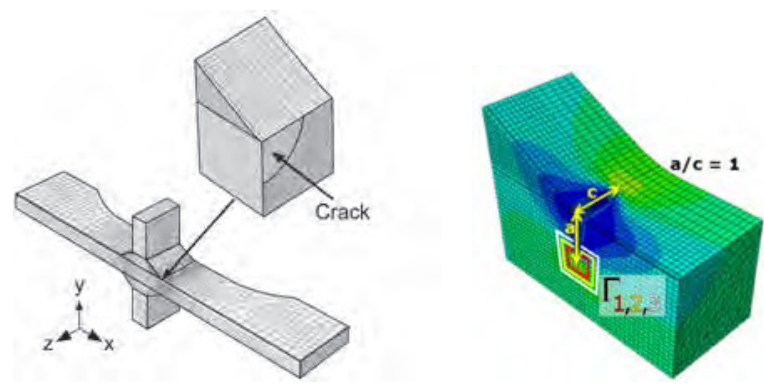

Fig. 2. FE modelling of a cruciform joint: the symmetry with respect to the mid-plane (parallel to the $x y$-plane) is exploited. A semi-circular crack $(a / c=1)$ is inserted at the weld toe, exactly at the position of maximum stress concentration.

The $\Delta J$-integral was always calculated at the deepest point of the crack front (crack depth). Examples of calculation paths are presented in Fig. 2 right. For a crack of length $a=0,5 \mathrm{~mm}$ and a stress range of $\Delta S=240 \mathrm{MPa}$ at $R=0$, the $\Delta J$-values obtained for the cruciform joint (by considering the elastic-plastic material behaviour) on the paths $\Gamma_{1}, \Gamma_{2}$ and $\Gamma_{3}$ (see Fig. 3 right) were respectively $0,330 \mathrm{~N} / \mathrm{mm}, 0,331 \mathrm{~N} / \mathrm{mm}$ and $0,334 \mathrm{~N} / \mathrm{mm}$, which means that $\Delta J$ can be considered as path independent (see Fig. 3a).

The influence of the nominal stress ratio on $\Delta J$ was studied, see Fig. 3b. As expected, the results show that the $R$-ratio has almost no influence on the $\Delta J$-integral as long as crack closure effects are not considered. 


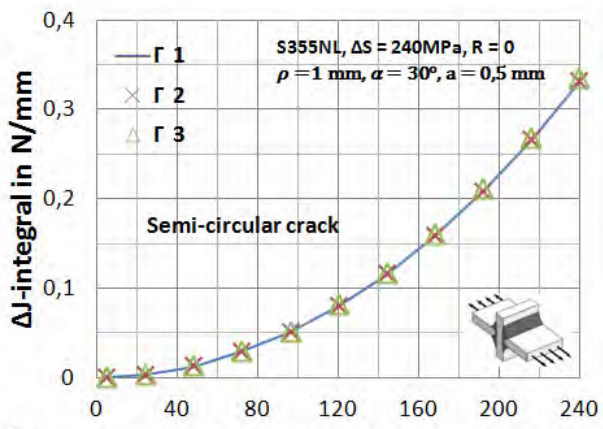

(a) Increment in nominal stress $\delta \mathrm{S}$ in $\mathrm{MPa}$

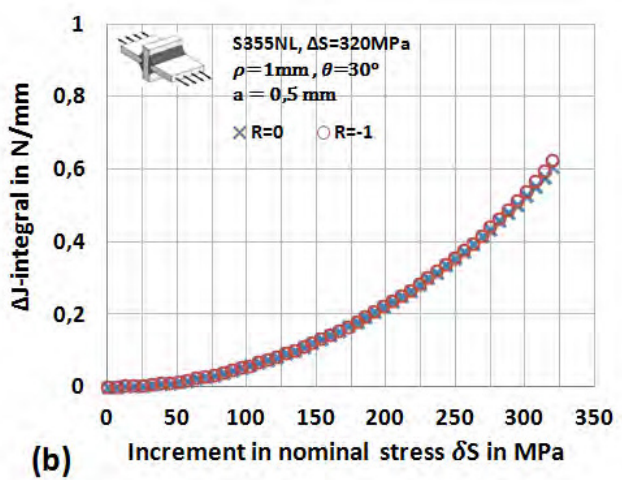

Fig. 3. (a)-Path-independency of the J-integral. (b)-Influence of the load ratio on the J-integral.

\subsection{Validation example}

For validation purposes, the finite element based $\Delta J$ results have been compared with those of an analytical model known as IBESS approach [21]. The approach is named after the IBESS-project, on which the present research took place. Zerbst et al. [21] calculated $\Delta K$ via weight functions and determine the $\Delta J$-integral by using the expression

$$
\Delta J=\frac{(\Delta K)^{2}}{\mathrm{E}^{\prime}} \cdot\left[f\left(\Delta L_{\mathrm{r}}\right)\right]^{-2} .
$$

Thereby, $f\left(\Delta L_{\mathrm{r}}\right)$ represents a plasticity correction function and depends on a cyclic $\Delta L_{\mathrm{r}}$ given as

$$
\Delta L_{\mathrm{r}}=\frac{\Delta \sigma_{\mathrm{app}}}{2 \cdot \sigma_{0}} .
$$

$\Delta \sigma_{\text {app }}$ corresponds to the applied cyclic stress (referring to the gross cross-section) and $\sigma_{0}$ is a reference yield stress, which was introduced to improve the accuracy of the estimate. For more details, a thorough description, including the parameter equations, is provided in [22].

Fig. 4 shows results obtained for a cruciform joint with semi-circular surface crack and a weld flank angle $\theta=30^{\circ}$. Elastic-plastic material behaviour was applied and the calculated $\Delta J$ values were transformed into "plasticity-corrected" $\Delta K_{p}$-values by using Eq. (13). A very good agreement can be observed between numerical results and the analytical model.

$$
\Delta K_{\mathrm{p}}=\sqrt{\Delta J \cdot E^{\prime}}
$$

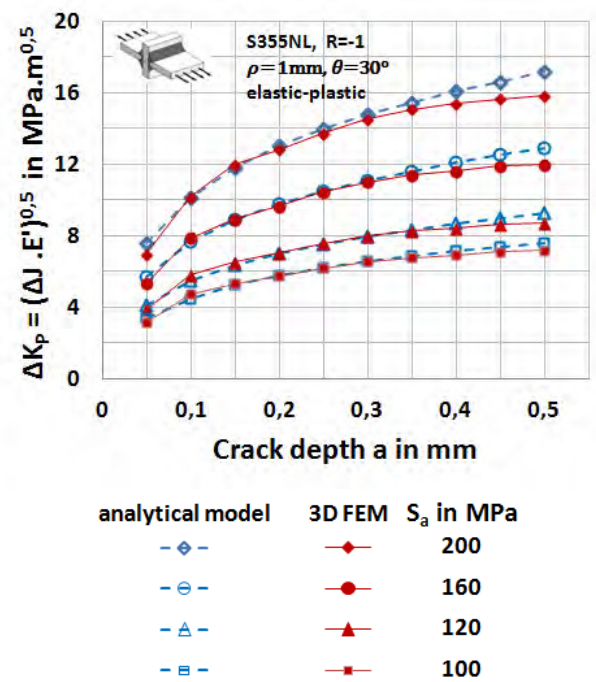

Fig. 4. Comparison between analytical and finite element based $\Delta K_{P}=\sqrt{\Delta J \cdot E^{\prime}}$ for cruciform joints with a flank angle of $\theta=30^{\circ}$. S355NL, $\rho=1 \mathrm{~mm}, a / c=1$.

\section{Fatigue crack growth simulations}

\subsection{Application of the node release technique to welded joints}

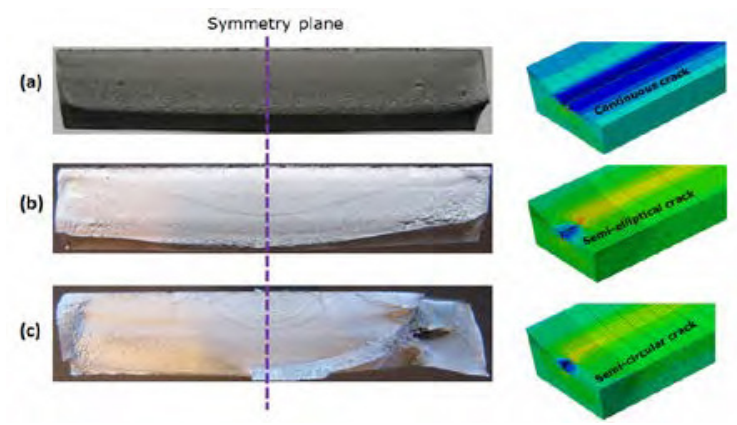

Fig. 5. Crack configurations: continuous crack (a); semielliptical crack (b); semi-circular crack (c).
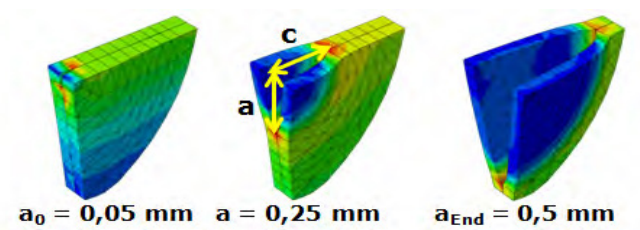

Fig. 6. Evolution of a semi-circular crack.

By assuming that the crack path and crack geometry are known in advance, the node release technique can be applied for the gradual extension of a crack from an initial crack length, $a_{0}$, up to a final crack length, $a_{\text {End }}$. In experiments, different types of crack geometry were observed for the joint: continuous, semi-elliptical as well as semi-circular cracks (see Fig. 5). Simulations that are carried out in this contribution only considered continuous (lead to shorter fatigue lives) and semicircular (lead to longer fatigue lives) cracks, i.e. $a / c \cong 0$ and $a / c=1$ respectively. Here, $a$ represents the crack depth and $c$ the half of the surface crack length. In Fig. 6 , the evolution of a semi-circular crack is shown in 
some configurations. An initial crack depth $a_{0}=$ $0,05 \mathrm{~mm}$ was selected, which approximately corresponds to the maximum defect size of 25 to $75 \mu \mathrm{m}$ that was measured in the specimens. These measurements were carried out by a project partner, see $[15,16]$. FE calculations were performed up to a final crack depth $a_{\text {End }}=0,5 \mathrm{~mm}$, which corresponds to the chosen numerical fracture criterion at short crack initiation. Only two load cycles were applied for every crack length configuration in order to restrict the FEcalculation time. In addition, two load cycles were applied before the first node release so that the residual stress relaxation/redistribution can be taken into account $[18,23]$. The crack extension was done at positive nominal stress (in order to satisfy the physical requirement that only an opened crack can propagate) and the increase in crack depth $\Delta a$ was chosen to be $0,05 \mathrm{~mm}$ for semi-circular cracks (3D calculations) and $0,01 \mathrm{~mm}$ for continuous cracks (modelled by using 2D models) respectively. The element length in the area of the crack was set equal to $\Delta a$. Note that the use of elements with linear shape functions is inevitable when contact constraints are applied.

\subsection{Algorithm of crack growth simulation}

The complete algorithm of crack growth simulation is presented in Fig. 7. This algorithm consists of 5 points:

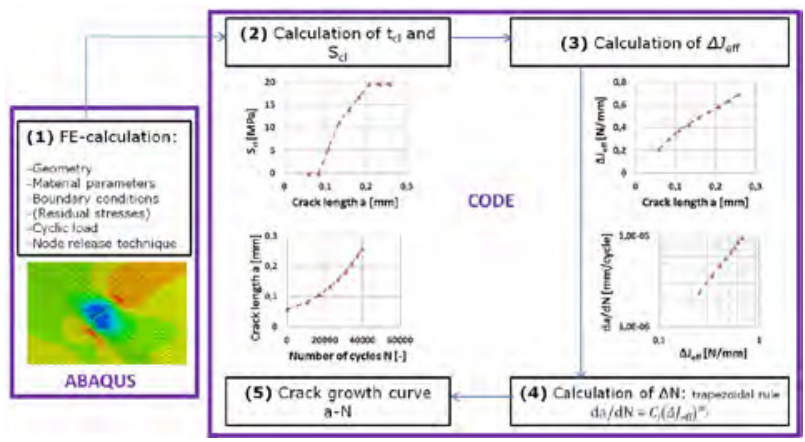

Fig. 7. Algorithm of crack growth simulation.

(1) FE-calculation: the complete structure calculation is carried out in a FE program (here ABAQUS) by using the "node release technique" to insert a crack and to extend it gradually until the final crack length (fracture criterion) is reached. The specimen geometry, material parameters, boundary conditions (including the gradual definition of contacts between the crack faces) and applied loads are needed here. If residual stresses are to be considered, they are inserted by using a procedure described by the authors in $[18,23]$.

(2) Identification of crack closure times $\left(t_{c l}\right)$ as well as crack closure stresses $\left(S_{\mathrm{cl}}\right)$ : after the FE calculation is done, the result file is accessed with a post-processing code in order to identify $\mathrm{t}_{\mathrm{cl}}$ and $S_{\mathrm{cl}}$ for every crack length configuration.

(3) Calculation of $\Delta J_{\text {eff: }}$ if the crack closure times are known, the effective cyclic $J$-integral $\Delta J_{\text {eff }}$ can be determined as function of the crack length.
(4) Calculation of $\Delta \mathrm{N}$ (number of load cycles which are needed to extend the crack from a length $a_{\mathrm{i}}$ to a length $\left.a_{\mathrm{i}+1}\right)$ : the crack growth equation,

$$
\frac{\mathrm{d} a}{\mathrm{~d} N}=C_{\mathrm{J}} \cdot\left(\Delta J_{\mathrm{eff}}\right)^{m_{\mathrm{J}}}
$$

is integrated by using the trapezoidal rule in order to determine $\Delta \mathrm{N}$ step by step from $a_{0}$ to $a_{\text {End }}$. The material parameters $C$ and $m$ of steel S355NL at $R=-1$ of the Paris equation,

$$
\frac{\mathrm{d} a}{\mathrm{~d} N}=C \cdot\left(\Delta K_{\mathrm{eff}}\right)^{m}
$$

were determined by a project partner [13]: $C=8,37$. $10^{-9} \mathrm{~mm} /$ cycl. $\left(\mathrm{MPa} \cdot \mathrm{m}^{1 / 2}\right)^{m}$ and $m=3$. These parameters were used for all calculations with steel S355NL at $R=-1$ as well as $R=0$.

By setting the crack propagation rate $\left(\frac{\mathrm{d} a}{\mathrm{~d} N}\right)$ equal in Eq. (14) and Eq. (15), and by using $K=\sqrt{J \cdot E^{\prime}}$ in case of linear elastic fracture mechanics, the following relationships can be identified for the determination of $C_{\mathrm{J}}$ and $m_{\mathrm{J}}$ :

$$
m_{\mathrm{J}}=\frac{m}{2}, \quad C_{\mathrm{J}}=C \cdot\left(E^{\prime}\right)^{m_{\mathrm{J}}} .
$$

Note that the calculated fatigue lives strongly depend on $C_{\mathrm{J}}$ and $m_{\mathrm{J}}$.

(5) Crack growth curve ( $a-N$ curve): the crack length is plotted together with the calculated number of cycles.

\section{Numerical results and discussions}

\subsection{Example of a cruciform joint made from steel S355NL}

Results obtained by using the procedure of crack growth simulation (see Fig. 7) are presented in this section. The selected crack geometry was semi-circular. The Chaboche's material parameters of steel S355NL were employed (see Table 1). Two different stress ratios $(R=0, R=-1)$ as well as three different stress amplitudes $\left(S_{\mathrm{a}}=120 \mathrm{MPa}, S_{\mathrm{a}}=160 \mathrm{MPa}\right.$ and $S_{\mathrm{a}}=$ $200 \mathrm{MPa}$ ) were used during computations.

The normalized crack closure stress, $S_{\mathrm{cl}} / S_{\max }$, and the crack closure function $U_{\text {eff }}$ (Eq. 17) are presented in Fig. 8. The function $U_{\text {eff }}$ determines the proportion of the load cycle in which the crack is opened and it is equal 1 for a fully opened crack or zero in case the crack is completely closed $\left(0 \leq U_{\text {eff }} \leq 1\right)$. In both functions $\left(S_{\mathrm{cl}} / S_{\max }\right.$ and $\left.U_{\mathrm{eff}}\right)$, a gradual build-up of crack closure effects can be observed. Two effects are to be distinguished: a transient crack closure behaviour that changes at the beginning of crack propagation with increasing crack length - this phenomenon only occurs at the short crack stage - and a stabilized crack closure behaviour that no longer changes with increasing crack length. This is typical for long cracks. It is also seen that the crack closure behaviour depends on the R-ratio: by 
maintaining the stress amplitude fixed, the functions $S_{\mathrm{cl}} / S_{\text {max }}$ and $U_{\text {eff }}$ increase with increasing $R$.

$$
U_{\mathrm{eff}}=\left(S_{\max }-S_{\mathrm{cl}}\right) /\left(S_{\max }-S_{\min }\right)
$$
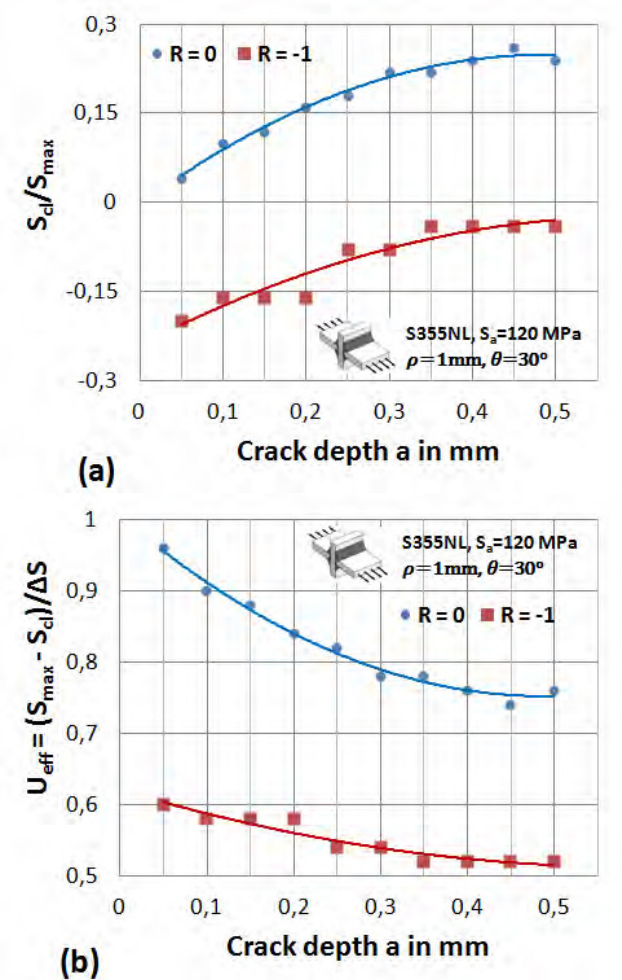

Fig. 8. Normalized crack closure stress (a) and crack closure function depending on the R-ratio (b), 3D-cruciform joint.
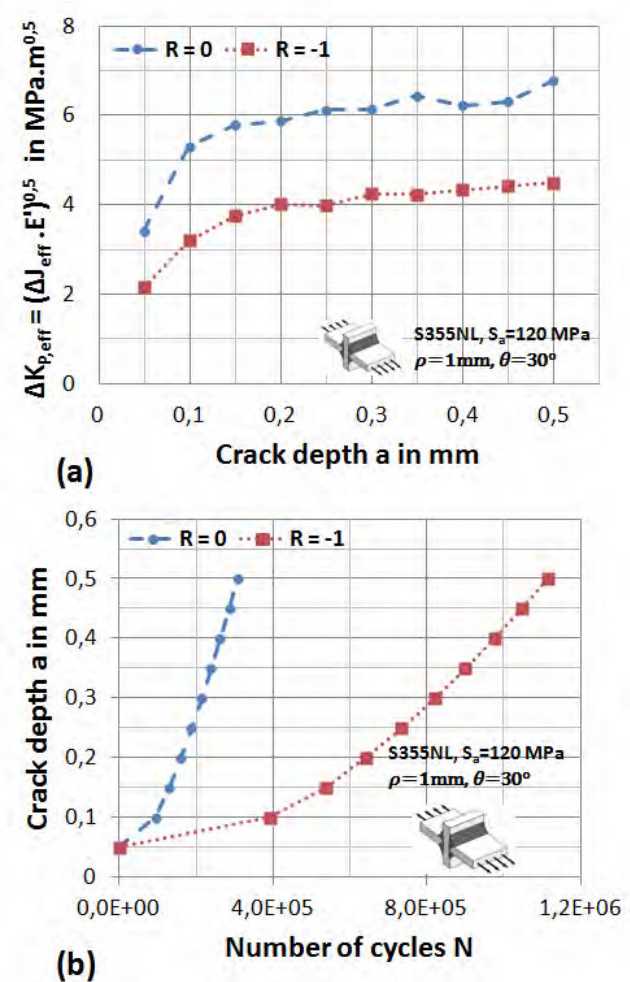

Fig. 9. Effective crack driving force $\Delta \mathrm{K}_{\mathrm{p}, \text { eff }}$ (a) and crack growth curve depending on the R-ratio (b).
In Fig. 9, the $\Delta K_{\mathrm{p}, \text { eff }}$-curve (effective value of the plasticity corrected range of the stress intensity factor, Fig. 9a) and the crack growth curve (Fig. 9b) are shown as function of the R-ratio. As expected, the effective crack driving force, $\Delta K_{\mathrm{p} \text {,eff }}$ increases with increasing crack depth as well as with increasing R-ratio. This also leads to the fact the fatigue life decreases with increasing $\mathrm{R}$ when the stress amplitude is kept constant. The sudden discontinuities in the numerical solutions (for example in Fig. 9a at $R=0$ and $a=0,35 \mathrm{~mm}$ ) may be due to plasticity in combination with variations of crack closure along the crack front or discontinuous crack closure. It should be mentioned that continuous functions were not fitted from this figure onward like in Fig. 8.

In Fig. 10 , the $\Delta K_{p, \text { eff }}$ curve as well as the crack growth curves are plotted as function of the nominal stress amplitude. As expected, $\Delta K_{p, e f f}$ increases with increasing stress amplitude. This leads to a decrease of the fatigue life when the stress amplitude increases.
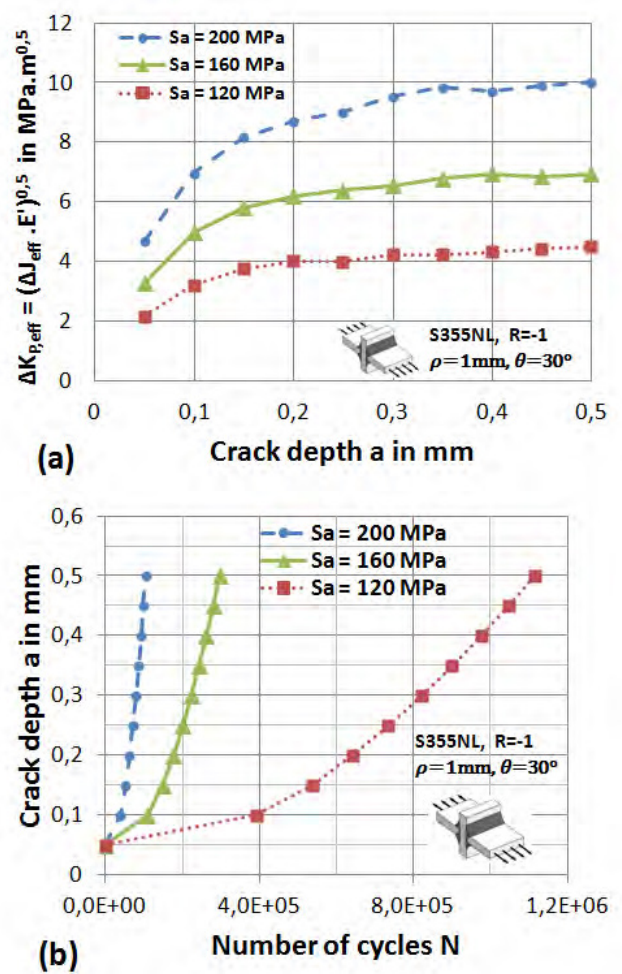

Fig. 10. Effective crack driving force $\Delta \mathrm{K}_{\mathrm{p} \text {,eff }}$ (a) and crack growth curve depending on the stress amplitude (b).

\subsection{Influence of residual stresses on the calculated fatigue lives}

The residual stress field that arises from the welding process can have a significant influence on the fatigue life [1, 18]. Tensile and compressive residual stresses were generated in the joint by means of structural calculations (see [24] for more details). The value of this stress at the critical weld notch was $+100 \mathrm{MPa}$ in case of a tensile residual stress field or $-100 \mathrm{MPa}$ in case of a compressive field. These stresses were then used for the numerical investigation of the residual stress influence on the fatigue crack growth. 

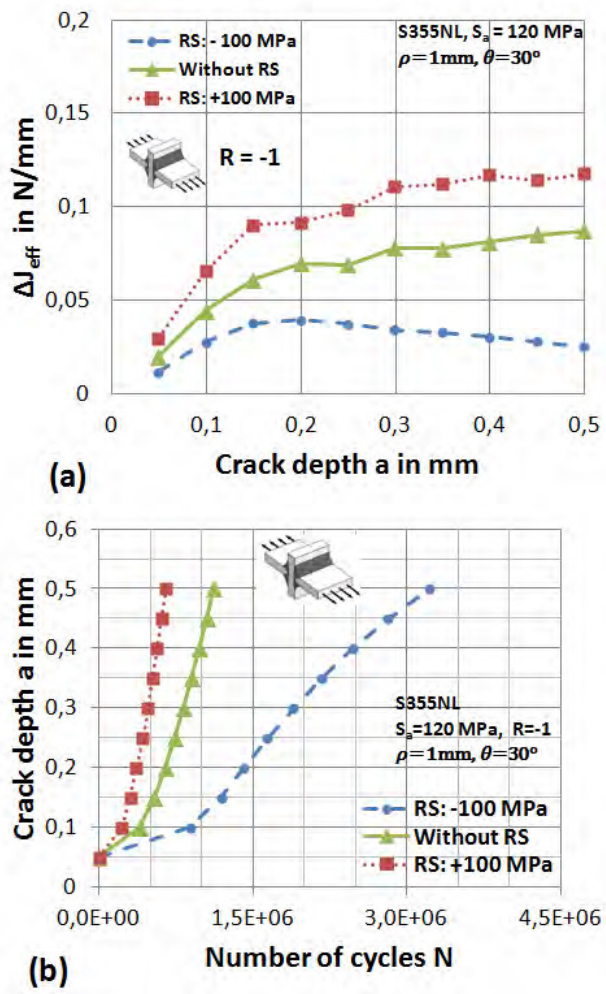

Fig. 11. Influence of residual stresses on $\Delta J_{\text {eff }}$ (a) and on the crack growth curve (b).

The effects of residual stresses on the effective cyclic $J$-integral, $\Delta J_{\text {eff, }}$ are illustrated in Fig. $11 \mathrm{a}$. As expected, tensile and compressive residual stresses lead to larger and smaller $\Delta J_{\text {eff }}$ values respectively, in comparison to $\Delta J_{\text {eff }}$ values that were calculated without residual stresses. The effects of residual stresses on the calculated fatigue life are illustrated in Fig. 11b. As expected, the consideration of tensile or compressive residual stresses leads to shorter or longer fatigue lives respectively. The reason for this is the fact that a compressive residual stress field increases crack closure effects, while a tensile residual stress field prevents these effects to occur.

\section{Comparison between numerical and experimental results}

In this Section, the fatigue lives at short crack initiation that were calculated for cruciform joints are compared with experimental results (see Fig. 12). Calculations were performed with and without residual stresses and the numerical fracture criterion at crack initiation was chosen to be $a=0,5 \mathrm{~mm}$. Experimental data were determined in various research groups, so that the experimental short crack initiation criterion depends on the method for short crack detection. The experimental fracture criterion at short crack initiation for S355NL was the appearance of small changes in the thermographic image [15]. This corresponds to a surface crack length of approximately $2 c=1 \mathrm{~mm}$.

Numerical and experimental results for cruciform joints S355NL in a stress relieved state (SR) are depicted in Fig. 12a. With regard to the different crack geometries (see Fig. 5), calculations were performed with 2Dmodels (corresponds to a continuous crack in 3D-model, which leads to shorter fatigue lives) as well as with 3Dmodels (for the case of a semi-circular crack, which leads to longer fatigue lives). Experimental fatigue life results almost lie between the $2 \mathrm{D}$ and $3 \mathrm{D}$ numerical fatigue life results, which represent the calculated boundaries. Thus, a good accordance between both results is achieved.
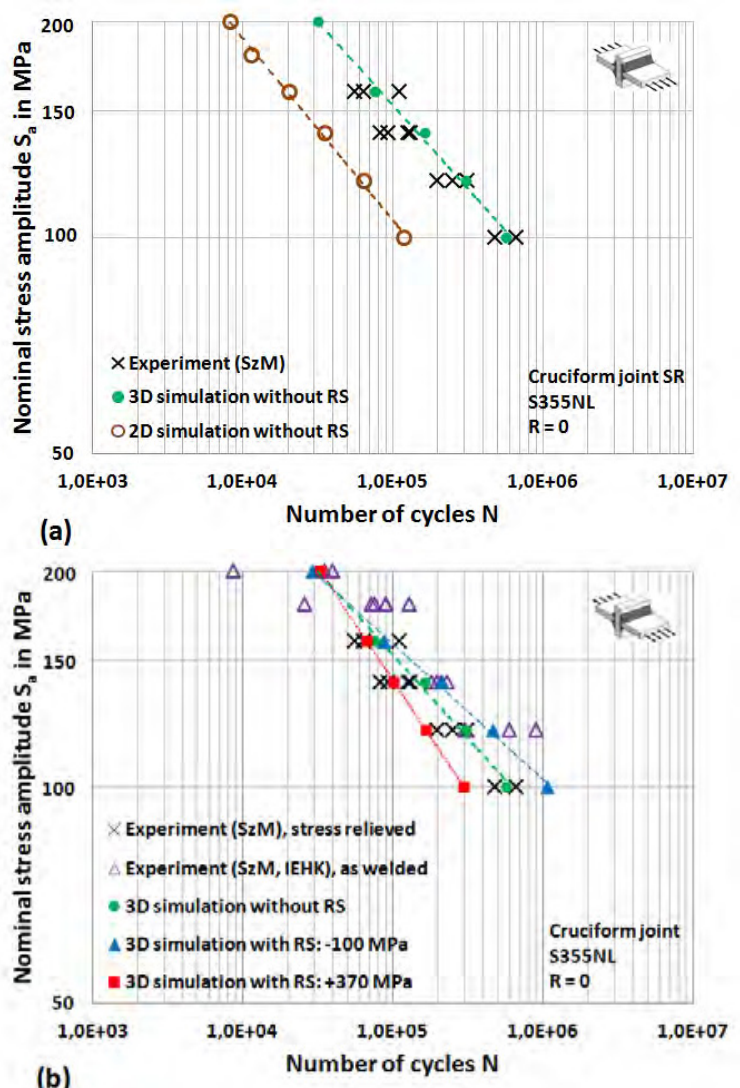

Fig. 12. Fatigue life at short crack initiation - comparison between numerical and experimental results. (a)-stress relieved (SR) specimens, (b)- stress relieved vs. as welded, $\mathrm{R}=0$.

The influence of compressive residual stresses and of high tensile residual stresses (equal to the yield stress) on the fatigue life was studied. Results are displayed in Fig.12b. Numerical results of computations with compressive residual stresses fit well to test results of aswelded specimens. A good accordance between experiments and calculations is achieved again. At lower stress amplitude, the stress relieved specimens lead experimentally to shorter fatigue lives compared to the as-welded specimens. This can be explained by the fact that according to residual stress measurements [23], the as-welded specimens exhibit compressive residual stresses, which lead to longer fatigue lives. At higher stress amplitude, both experimental and numerical results show almost no influence of welding residual stresses on the fatigue life.

Calculations with high tensile residual stresses (equal to the yield stress) lead to conservative lifetimes at lower amplitudes. Generally, in a case where the 
welding residual stress field is unknown, it is often recommended in the literature [25] to use high tensile residual stresses for the fatigue life prediction. Therefore, the conservative effect of such an approach for the consideration of welding residual stresses is confirmed.

\section{Summary and conclusion}

Fatigue crack growth simulations have been performed by taking into consideration plasticity induced crack closure effects as well as the effects of welding residual stresses. For this purpose, an algorithm of fatigue life prediction was presented. Thereby, the effective cyclic $J$ integral $\left(\Delta J_{\text {eff }}\right)$ was used as crack tip parameter in a relation similar to the Paris equation for the calculation of the fatigue life. Analyses were performed with two and three dimensional FE models by using the node release technique together with the $\mathrm{FE}$ program ABAQUS.

Crack closure functions and the effective crack driving force ( $\Delta J_{\text {eff }}$ or $\left.\Delta K_{\mathrm{p}, \text { eff }}\right)$ were determined numerically for surface cracks in butt and cruciform joints. Numerical results show that the closure functions and the effective crack driving force strongly depend on the load ratio as well as the load amplitude. It was possible to describe the transient crack closure behaviour that occurs at the short crack stage as well as the stabilized crack closure behaviour for long cracks.

The numerical fatigue life results were compared with experimental data: a good accordance between numerical and experimental results was achieved for both stress relieved and as-welded specimens. Thus, $\Delta J_{\text {eff }}$ is obviously an appropriate parameter for calculations concerning fatigue life prediction in welded joints.

The influence of welding residual stresses on the fatigue life was investigated numerically as well as experimentally. As expected, numerical results show that at lower stress amplitude, a compressive residual stress field is favourable to the fatigue life, while a tensile residual stress field is unfavourable. The effect of residual stresses can be neglected only for large load amplitudes. Experimental observations show that the fatigue lives of the stress relieved specimens are smaller than those of the as-welded specimens. This was due to the fact that the as-welded specimens exhibit compressive residual stresses (according to residual stress measurements), which are favourable to the fatigue life.

The German Research Foundation (Deutsche Forschungsgemeinschaft) is greatly acknowledged by the authors for financing this project under grant no. V0729/14-1.

\section{References}

1. C.M. Sonsino, Int. J. Fatigue 31, pp. 88-101, (2009)

2. D. Tchoffo Ngoula, H. Th. Beier, M. Vormwald, DVS-Bericht 315, pp. 294-299, (2015)
3. W. Elber, Engng Fract Mech 2, pp. 37-45 (1970)

4. H.A. Richard, M. Sander, 3. erweiterte Auflage, Springer Vieweg, Wiesbaden (2012)

5. W. Fricke, Mar. Struct. 16, pp. 185-200, (2003)

6. D. Radaj, C.M. Sonsino, W. Fricke, Int. J. Fatigue 31, pp. 2-11 (2009)

7. S.J. Maddox, Metal Const. 2, pp. 327-331, (1970)

8. J. Polak, Comprehensive Structural Integrity, 4, 1-39 (2003)

9. N.E. Dowling, J.A. Begley, ASTM STP 590, 82-103 (1976)

10. J.R. Rice, ASME J. Appl. Mech., 35, 376-386, (1968)

11. McClung, R.C., Chell, G.G., Lee, Y.D., Russel, D.A. and Orient, G.E, Report NASA/CR-1999209428, Marshall Space Flight Center, 1999.

12. Vormwald, M., In Radaj, D. and Vormwald, M., Springer, Heidelberg, p. 391-481, 2013.

13. U. Zerbst: Schussbericht zu den IGF-Vorhaben 17518 N/1 , IBESS-A3, 2016.

14. Tchoffo Ngoula, D., Beier, H. Th., Vormwald, M.: Abschlussbericht zu den DFG-Vorhaben V0729/14-1, IBESS-G3, 2016.

15. H. Th. Beier, B. Schork, J. Bernhard, D. Tchoffo Ngoula, T. Melz, M. Oechsner, M. Vormwald, Mat.-wiss. U. Werkstofftech. 47, No. 10, (2016)

16. B. Schork, U. Zerbst, P. Kucharczyk, J. Bernhard, M. Kaffenberger, J. Baumgartner, M. Oechsner, DVS-Bericht 315, pp. 271-276, 2015.

17. J.L. Chaboche, Int. J. Plast. 24, 1642-1693, 2008.

18. Tchoffo Ngoula, D., Beier, H. Th., Vormwald, M., Int. J. Fatigue 101(2), pp. 253-262, 2017.

19. de Matos, P.F.P., Nowell, D., Engineering Fracture Mechanics, 75, pp. 2087-2114, 2008.

20. Tchoffo Ngoula, D., Madia, M., Beier, H. Th., Vormwald, M., Zerbst, U., Engng Fract Mech , 2017.

http://dx.doi.org/10.1016/j.engfracmech.2017.06 .023 .

21. Zerbst, U., Madia, M., Hellmann, D., Engng. Fracture Mech. 82, 115-134, 2011.

22. Madia, M., Zerbst, U., Beier, H.Th. and Schork, B., Engng Fract Mech , 2017. http://dx.doi.org/10.1016/j.engfracmech.2017.08 .033 .

23. Hensel, J., Nitschke-Pagel, T., Tchoffo Ngoula, D., Beier, H.-Th., Tchuindjang, D. and Zerbst, U., Engng Fract Mech , 2017. $\underline{\text { http://dx.doi.org/10.1016/j.engfracmech.2017.10 }}$ .024 .

24. Tchoffo Ngoula, D., Rep 117, Inst Stahlbau Werkstoffmech, TU Darmstadt, 2018.

25. Hobbacher, F. (2016), Second Edition, IIW Document IIW-2259-15, Springer. 